\title{
Development of inner tracking systems equipped with CMOS pixel sensors for future collider experiments
}

\author{
Isabelle Ripp-Baudot ${ }^{* \dagger}$ \\ IPHC Strasbourg, CNRS/IN2P3, Université de Strasbourg, France. \\ E-mail: rippein2p3.fr
}

\begin{abstract}
The physics goals of a growing number of subatomic physics experiments assume vertexing and tracking performances calling for a specific pixel technology, which offers a high data rate capability as well as precision, low power and cost standards departing from those of the most widely used devices. CMOS Pixel Sensors (CPS) address this challenge and have already been chosen to equip operating devices. This paper shows that the capability of CPS to comply with more ambitious requirements of future experiments relies on a recently addressed CMOS process, based on a $0.18 \mu \mathrm{m}$ feature size and a high resistivity epitaxial layer of sizeable thickness. Prototype sensors exploring the process detection performances were extensively tested with beam particles. An overview of their measured performances is provided and the development strategy to reach real size sensors with full functionality is described.
\end{abstract}

The European Physical Society Conference on High Energy Physics -EPS-HEP2013

18-24 July 2013

Stockholm, Sweden

\footnotetext{
* Speaker.

$\dagger$ on behalf of the PICSEL group, IPHC Strasbourg: http://www.iphc.cnrs.fr/Research-team,105.html
} 


\section{Introduction}

CMOS pixel sensors (CPS) are monolithic devices where the signal sensing and analogue treatment are integrated within the same chip. Details on the CPS principle of operation are provided in [1]. Their prominent features consist in their very high granularity associated to a low material budget, offering together the possibility to reconstruct decay vertices of short living and low momentum particles with unprecedented precision. Nevertheless, despite their wide potential for charge particle tracking, applications of CPS in high energy experiments was so far limited by the available CMOS technologies. The latter exploit the fabrication processes used in industry for mass production of integrated circuit, with hence very good fabrication yields and low cost, and furthermore the benefit from a continuous technological evolution.

The first generation of CPS used in subatomic physics was essentially relying on a $0.35 \mu \mathrm{m}$ CMOS process. In order to address the requirements of upcoming experiments, a process with smaller feature size $(0.18 \mu \mathrm{m})$ was chosen, with the benefit of a larger number of metal layers, the possibility to use both types of transistors inside the pixels and an epitaxial layer of increased thickness and resistivity. This paper presents the assessment of the performances of this CMOS technology for charged particle tracking and discusses its potential.

The state-of-the-art MIMOSA sensor equipping currently operating devices, based on a 0.35 $\mu \mathrm{m}$ technology, is described in the next section (section 2). Performance improvements required to equip inner tracking systems of future projects are discussed in section 3 and the development path to match these specifications is presented in section 4 , relying on the $0.18 \mu \mathrm{m}$ process mentioned above. Section 5 describes the new MIMOSA prototypes manufactured in this technology and presents preliminary characterisation results. Finally, section 6 concludes the paper and provides an outlook.

\section{State-of-the-art CPS}

CPS presently used in tracking layers in high energy physics are from the MIMOSA series, developed at IPHC Strasbourg [2] and based on a $0.35 \mu \mathrm{m}$ OPTO process. The state-of-the-art CPS is represented by the MIMOSA-26 sensor [3] of the beam telescope from the EU/FP-6 project EUDET [4] operating since 2008, and more recently by the MIMOSA-28 sensor (also known as ULTIMATE) equipping the upgraded vertex detector (called PXL) of the STAR experiment at RHIC [5]. The PXL detector was partially installed in May 2013 and successfully commissioned during one full month of run within the STAR experimental setup.

These sensors are thinned down to $50 \mu \mathrm{m}$ and their sensitive layers are only partially depleted. Sensors are very granular, with pixel dimensions of $20.7 \times 20.7 \mu \mathrm{m}^{2}$ in a total active area of $19.9 \times 19.2 \mathrm{~mm}^{2}$ in STAR, resulting in a matrix of $\sim 0.9$ millions of sensing nodes. The architecture has been chosen to preserve the CMOS pixel sensors prominent assets, which are the high granularity together with the low material budget. As the latter point assumes also a low power dissipation to avoid active cooling, a column-parallel read-out has been chosen (referred to as the rolling-shutter read-out), powering only one row at a time with a read-out frequency of $5 \mathrm{MHz}$ (corresponding to $200 \mathrm{~ns} / \mathrm{row}$ ). Amplification and correlated double sampling (CDS) are performed within each pixel, and each column of pixels is terminated with a discriminator [6], 
which output is processed through a zero suppression logic [7]. This architecture is well adapted to process a hit rate exceeding $10^{6} \mathrm{hits} / \mathrm{cm}^{2} / \mathrm{s}$, with a power dissipation kept around $150 \mathrm{~mW} / \mathrm{cm}^{2}$, enabling using an easy-to-implement air-flow cooling. The detection performances of MIMOSA28 with respect to STAR specifications were assessed with a $120 \mathrm{GeV} \pi$ beam produced at the CERN-SPS. In particular the detection efficiency is nearly $100 \%$ associated to a low fake hit rate below $10^{-5}$ with a coolant temperature of $30^{\circ} \mathrm{C}$. The measured single point resolution is $\gtrsim 3.5 \mu \mathrm{m}$, achieved with binary charge encoding. Finally the radiation tolerance is validated up to a fluence of $3 \times 10^{12} \mathrm{n}_{e q} / \mathrm{cm}^{2}$ and an ionising dose of $150 \mathrm{kRad}$ at $30^{\circ} \mathrm{C}$.

\section{Future experiments performance requirements}

Today CPS are considered as candidate pixel sensors for future experiments or detector upgrades. In particular they have been chosen as the baseline option to upgrade the ALICE Inner Tracking System (ITS) [8] at LHC. They are furthermore investigated for several other projects, their performances being in general relevant for the needs of $e^{+} e^{-}$and heavy ions experiments. They are studied to equip vertex detectors of the CBM experiment at FAIR [9], of the ILD at a future ILC [10] and to introduce a new inner tracker in the BES-3 experiment at BEPC. Specifications of such experiments' inner trackers are governed by the same kind of constraints. The latter are driven on one hand by physics goals calling for particularly good vertexing performances (good single point resolution and low multiple scattering) and on the other hand by an experimental environment characterised by high particle rates and moderate radiation loads if compared to the LHC. Table 1 summarises the required performances of the current operating detector equipped with CPS in STAR and how they have to improve in order to match specifications of future projects.

\begin{tabular}{|c|c|c|c|c|c|}
\hline experiment & spatial resolution & read-out time & TID & Fluence & $\mathrm{T}_{\text {coolant }}$ \\
\hline STAR & $\sim 5 \mu \mathrm{m}$ & $\sim 200 \mu \mathrm{s}$ & $150 \mathrm{kRad}$ & $3 \times 10^{12} \mathrm{n}_{e q} / \mathrm{cm}^{2}$ & $30^{\circ} \mathrm{C}$ \\
\hline future projects & $3-5 \mu \mathrm{m}$ & $1-30 \mu \mathrm{s}$ & $\leq 10 \mathrm{MRad}$ & $\leq 10^{14} \mathrm{n}_{e q} / \mathrm{cm}^{2}$ & $<0-30{ }^{\circ} \mathrm{C}$ \\
\hline
\end{tabular}

Table 1: Required performances of the current operating detector equipped with CPS in STAR and how they have to improve in order to match specifications of future projects (TID stands for Total Ionising Dose).

Required read-out times are estimated here by considering hit rates, trigger rates and data flows. However, these specifications are strongly correlated with each other. For instance if the required read-out time results from occupancy rate considerations, due to high machine-induced background rates (directly proportional to the sensor integration time), then a finer segmentation may help relaxing this constraint. Moreover, specifications required for the innermost layers depend on the quality (spatial resolution and time-stamp) of the extrapolated track, reconstructed with all outer tracker layers. Therefore, a global design of the inner tracking system geometry enables to go beyond current technology limits.

Nevertheless, the conclusion of these values is that the next generation of experiments calls for pixellated sensors with higher read-out speed and better radiation hardness. And this breakthrough in performances has to be achieved without decreasing the segmentation or increasing the power consumption.

Furthermore, the ALICE collaboration has shown through the design study performed to upgrade its Inner Tracking System [8], that better tracking performances were reached with 7 layers 
equipped with CMOS pixel sensors rather than only the 3 innermost layers being pixellated and the 4 outermost layers being equipped with double-sided silicon strip sensors. In particular the tracking efficiency and the momentum resolution are significantly better with the all-pixel option.

Two particular constraints have to be taken into account when considering equipping large radius layers with pixel sensors: the cost of the full device (corresponding to $\sim 10 \mathrm{~m}^{2}$ in ALICE), not only sensors but also service tasks and, if needed, read-out electronics, and the possibility to optimise differently the detection performances of inner and outer layers. Indeed, because of occupancy rate and spatial resolution considerations, high granularity has to be favoured at small radii, hence harming generally either the read-out time or the power consumption. Such specifications may be relaxed at larger radii for the benefit of power consumption.

\section{Exploiting the evolution of the CMOS technology}

One particular difficulty with monolithic planar technologies is that a single process determines as well the sensing as the signal performances. However, fabrication parameters are not optimised for charged particles detection but obviously for market considerations. The choice of the process used to design a tracking sensor for high energy physics experiments may be governed by epitaxy characteristics (doping profile) to ensure a good signal amplitude, at the expense of the front-end electronics circuitry parameters (feature size, number of metal layers), or vice versa. As a consequence, the real potential of CMOS technology for charged particles tracking has not been fully exploited yet. These considerations drive the 3D CMOS technology developments [11] for high energy physics, which is a way to optimise separately sensing performances and signal processing by using 2 different processes for each tier.

Another limitation is that the use of P-MOS transistors within the sensitive area is generally restricted because of parasitic charge collection originating from the $\mathrm{N}$-wells hosting this type of transistors. This limitation, in addition to the feature size, implies that most of the signal processing is performed on the sensor periphery in MIMOSA up to now, resulting in a read-out speed of about $100 \mu \mathrm{s}$.

The breakthrough needed to overcome these limitations has been enabled by the availability of a $0.18 \mu \mathrm{m}$ imaging technology offering a burried P-well option together with a high resistivity epitaxy. Taking benefit from the opportunity opened by this technology, a strategy to improve the sensors' radiation hardness and read-out speed while keeping the power dissipation low has been set up. First, smaller feature size and higher epitaxial resistivity is expected to further improve the radiation hardness. Moreover, functionalities within the pixel array can be enhanced thanks to smaller feature size and larger number of metal layers, while the power consumption is kept under control thanks to in-pixel digitisation so that only binary signals are carried out to the sensor periphery. In this way, the row read-out is fasten to $100 \mathrm{~ns}$ instead of $200 \mathrm{~ns} / \mathrm{row}$ without in-pixel digitisation. Another way to further improve the read-out speed, which is not related to the smaller feature size, consists in subdividing the sensor in sub-arrays to read more pixel rows at a time. Pixel dimensions can also be increased, in order to decrease the number of pixels composing a read-out unit (e.g. a column). Pixels may be enlarged, on one hand, along the column direction, to decrease the number of rows to be read through rolling-shutter, resulting in a shorter sensor read-out time. They may also be enlarged, on the other hand, parallel to the row direction, to decrease the number 
of pixels per row and hence mitigate the power consumption. This flexibility is a key asset in the perspective of equipping large surfaces at large radii.

The possible resulting degradation of the spatial resolution due to enlarged pixel dimensions is addressed through several considerations:

- It has to be mentioned that the pixel dimensions of MIMOSA sensors are particularly small, typically of the order of $20 \times 20 \mu \mathrm{m}^{2}$, with respect to usual dimensions of other pixel sensors in high energy experiments, varying between $\sim 55 \times 55 \mu \mathrm{m}^{2}$ [12] and $400 \times 50 \mu \mathrm{m}^{2}$ [13]. The choice of the pixel dimension results from a global parameter optimisation. For instance for outer layers at large radius, where the hit rate is usually lower and the spatial resolution requirements are relaxed with respect to inner layers, it may be more relevant to decrease the power consumption at the expense of the segmentation but for the benefit of material budget.

- The ultra-low material double-sided PLUME ladder [14], equipped on both sides with CPS, provides 2 measurement points on sensors separated by $2 \mathrm{~mm}$. This spatial proximity allows to match these 2 hits and to associate them efficiently with the track reconstructed in outer layers and extrapolated on PLUME, even in case of moderate spatial resolution of the hits measured with PLUME. One particular advantage of this approach is the possibility to equip one side of the ladder with sensors optimised for spatial resolution (with small pixels, at the expense of a high read-out time to limit the power consumption) and the other side with sensors designed with emphasis on the time resolution (enlarged pixels and faster read-out time). Such a device provides finally the equivalent of one hit measured with good spatial and time resolution.

- Finally a small grid size enables to perform in-pixel digitisation. For instance a 3-bit ADC may be implanted in a pixel of dimension $40 \times 40 \mu \mathrm{m}^{2}$ with an expected resulting spatial resolution better than $3 \mu \mathrm{m}$.

\section{Characterisation of new CMOS pixel sensor prototypes}

End of 2011 and in 2012, several MIMOSA-32 prototype chips were manufactured in the 0.18 $\mu \mathrm{m}$ technology mentioned earlier, based on a $18 \mu \mathrm{m}$ thick, high resistivity ( $>1 \mathrm{k} \Omega . \mathrm{cm}$ ), epitaxial layer. It is a quadruple well process with deep P-type skins, embedding N-wells hosting P-MOS transistors. The prototypes were subdivided in several small matrices where various pixel designs were implemented. Pixel dimensions of $20 \times 20,20 \times 33,20 \times 40$ and $20 \times 80 \mu \mathrm{m}^{2}$ are explored, as well as various charge collection and amplification systems, with sensing diode surfaces of 9 and $11 \mu \mathrm{m}^{2}$ and N-MOS and P-MOS transistor-based amplifiers. Each sub-matrix features a read-out time of $32 \mu \mathrm{s}$.

These sensors were tested on one hand in the laboratory with an ${ }^{55} \mathrm{Fe}$ source, looking at the chip response to the $5.9 \mathrm{keV} \mathrm{X-Rays} \mathrm{emitted,} \mathrm{and} \mathrm{on} \mathrm{the} \mathrm{other} \mathrm{hand} \mathrm{with} \mathrm{high} \mathrm{energy} \mathrm{pions} \mathrm{beams}$ at the CERN-SPS, looking at the chip detection performances of particles near the minimum ionisation energy. These tests aimed at comparing the charge collection efficiencies of the different pixel designs and at assessing the radiation tolerance of the new technology, as these properties are 
difficult to predict with simulations. About 90 points were measured in total, with air cooling temperatures between $15^{\circ} \mathrm{C}$ and $30^{\circ} \mathrm{C}$, and different irradiations up to a combined radiation load of 1 MRad and $10^{13} \mathrm{n}_{e q} / \mathrm{cm}^{2}$ corresponding to the ALICE upgraded Inner Tracker System requirements.

First results of these tests have already been presented in [15]. The main conclusion is the efficient charge collection, guaranteeing a detection efficiency above $99 \%$ for square pixels after a combined radiation load of $1 \mathrm{MRad}$ and $10^{13} \mathrm{n}_{e q} / \mathrm{cm}^{2}$ at $30^{\circ} \mathrm{C}$. A slight noise increase is observed with increasing dose, as expected due to leakage current. But the signal-over-noise ratio (SNR) remains high after the highest combined radiation load considered here, with a maximum probability value above 20 for all squared pixel dimensions at $15^{\circ} \mathrm{C}$, as illustrated on figures 1 and 2 .

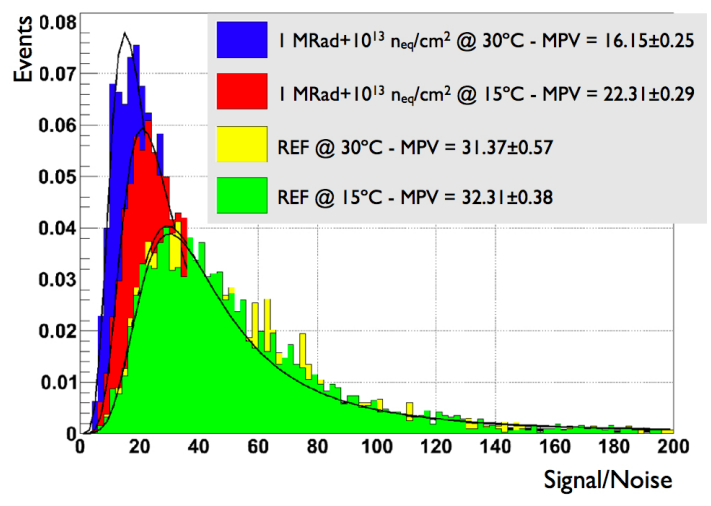

Figure 1: Observed response of MIMOSA-32 $\left(20 \times 20 \mu \mathrm{m}^{2}\right.$ pixels $)$ to $\sim 100 \mathrm{GeV}$ pions. The figure shows the signal over noise ratio (SNR) distribution before irradiation and after a combined load of $1 \mathrm{MRad}$ and $10^{13} \mathrm{n}_{e q} / \mathrm{cm}^{2}$ at different air-cooling temperatures.

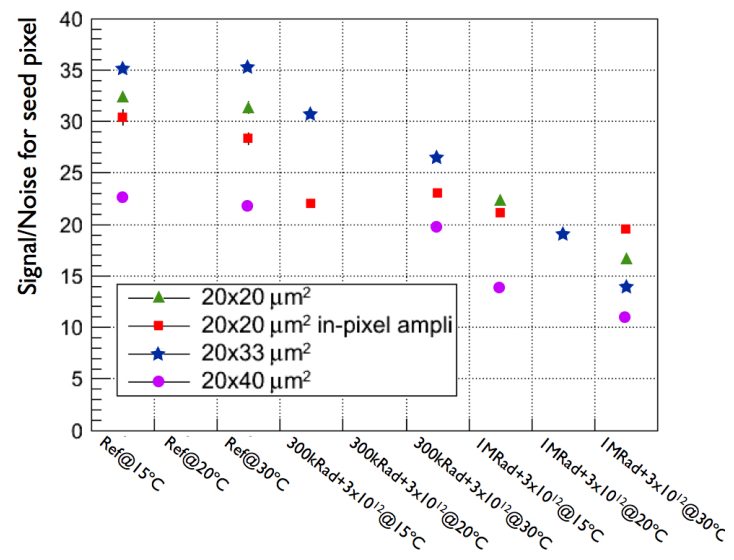

Figure 2: Signal over noise ratio evolution of different MIMOSA-32 pixel designs with the ionising and non-ionising radiation loads and different air-cooling temperatures.

Beam test analogue data are used to simulate off-line the binary charge encoding and the signal discrimination which will be performed with the final sensor. A common cut on SNR is applied for all pixels, allowing to evaluate the single point resolution and the detection efficiency as a function of the applied discriminator threshold. Thanks to signal sharing between pixels, a single point 
resolution of $3.2 \pm 0.2 \mu \mathrm{m}$ is measured for $20 \times 20 \mu \mathrm{m}^{2}$ pixels. In case of $20 \times 40 \mu \mathrm{m}^{2}$ elongated pixels with a staggered sensing diode network, the measured single point resolution is $5.4 \pm 0.2 \mu \mathrm{m}$ in both directions.

\section{Conclusion and outlook}

CMOS pixel sensors based on a $0.35 \mu \mathrm{m}$ technology are now a mature technology and are already equipping successfully operating devices. Today CPS are also considered as candidate sensors for future projects at $e^{+} e^{-}$and heavy ions colliders. Their requirements are being addressed with a more advanced CMOS process based on a $0.18 \mu \mathrm{m}$ feature size. Its fabrication parameters, which include 6 metal layers and a high resistivity epitaxial layer of sizeable thickness, and allow for both transitor types inside the pixel array, offer new perspectives in charged particle tracking. It extends in particular the use of CPS to pixellated trackers of several squared meters, like the upgraded ALICE-ITS. Satisfactory charge collection performances and radiation hardness were obtained with several prototype sensors manufactured in this $0.18 \mu \mathrm{m}$ technology exposed to high energy particle beams. These results allow moving to the next steps of the development which target in particular a decrease of the integration time down to $\lesssim 20 \mu$ s in 2016 while limiting the power consumption below $100 \mathrm{~mW} / \mathrm{cm}$, in order to comply with the ALICE upgraded ITS specifications. This evolution may ultimately allow to nearly fully exploit the assets of the CPS technology and achieve a read-out time close to $1 \mu \mathrm{s}$ in rolling shutter mode and a radiation hardness approaching $10 \mathrm{MRad}$ and $10^{14} \mathrm{n}_{e q} / \mathrm{cm}^{2}$.

\section{References}

[1] R. Turchetta et al., Nucl. Instrum. Meth. A 458 (2001) 677-689.

[2] http://www.iphc.cnrs.fr/PICSEL

[3] J. Baudot et al., IEEE Nucl. Sci. Symp. Conf. Rec. 2009 (2009) 1169-1173.

[4] The EUDET Consortium, arXiv:1201.4657, January 2012.

[5] L. Greiner et al., Nucl. Instrum. Meth. A 650 (2011) 68-72.

[6] Y. Degerli et al., Nucl. Instrum. Meth. A 602 (2009) 461-466.

[7] A. Himmi et al., proceedings of TWEPP'09, 2009, September 21-25, Paris.

[8] The ALICE Collaboration, CERN-LHCC-2012-013, Sept. 11, 2012.

[9] M. Deveaux et al., PoS Vertex2008 (2008) 028.

[10] Y. Voutsinas et al., PoS Vertex2012 (2013) 025

[11] R. Yarema et al., JINST 8 (2013) C01052.

[12] K. Akiba et al., Nucl.Instrum.Meth. A661 (2012) 31-49.

[13] The ATLAS Collaboration, G. Aad et al., JINST 3 (2008) P07007.

[14] http://www.iphc.cnrs.fr/PICSEL/PLUME.html

[15] J. Baudot et al., arXiv:1305.053, proceedings of the 13th Vienna Conference on Instrumentation (VCI 2013), 11-15 Feb 2013, Vienna, Austria. To be published in Nucl. Instrum. Meth. A. 